Bull. Mater. Sci., Vol. 18, No. 5, September 1995, pp. 469-495. (C) Printed in India.

\title{
Electrically conducting polymers: from fundamental to applied research
}

\author{
A K BAKHSHI \\ Department of Chemistry, Panjab University, Chandigarh 160014, India \\ MS recelved 13 March 1995
}

\begin{abstract}
A review of the state of the art of electrically conducting polymers is presented. Special emphasis is laid on the nature of the doping processes which induce high electrical conductivity in conjugated organic polymers and on the nature of charge carriers such as solitons, polarons and bipolarons produced in them as a result of such processes. Various factors affecting the electrical conductivity of these polymers and the various models proposed to explain charge transport in them are also discussed. The present status of the structure-property relationship in these conducting polymers and the various routes currently pursued to tailor novel conducting polymers are also briefly mentioned. Finally the various applications of these novel electronic materials are described.
\end{abstract}

Keywords. Charge carriers; crystal orbitals; ladder polymers; electrical conductivity; batteries.

\section{Introduction}

The advent of polymers (plastics) represents one of the important industrial revolutions of the 20th century. Polymers with properties such as strength, elasticity, plasticity, toughness and frictional resistance, often comparable with those of metals, offer a lot of very important advantages over the latter such as lighter weight, greater workability, resistance to corrosion and lower cost. As a result these materials have not only replaced metals in many areas of applications but have also infiltrated our day to day life with a wide range of products extending from most common consumer goods to highly specialized applications in space and aeronautics.

There is, however, one important fundamental property viz. electrical conductivity which distinguishes metals from polymers. Its value for metals is very high and is generally of the order $10^{4}-10^{6} \mathrm{~S} \mathrm{~cm}^{-1}$ (good conductors such as copper and silver have conductivities close to $10^{6} \mathrm{~S} \mathrm{~cm}^{-1}$ ) while for polymers which are generally insulators this value does not exceed $10^{-14} \mathrm{~S} \mathrm{~cm}^{-1}$ (good insulators such as teflon and polystyrene have conductivity values close to $\left.10^{-18} \mathrm{~S} \mathrm{~cm}^{-1}\right)$. Though the low electrical conductivity of polymers has found its immense use in the manufacture of insulators and dielectric substances, the question of producing polymers which exhibit conductivity similar to that of metals has always engaged researchers. The effort in this direction got a boost when Little (1964) proposed the possibility of room temperature superconductivity in materials consisting of a conducting backbone to which polarizable sidegroups are attached. A few years later, many organic crystals with high conductivity were discovered and some of them did show superconductivity at low temperatures. These crystals often consist of stacks of donor and acceptor molecules and they display metallic conductivity when the separation between the molecules along the stack is small. Perpendicular to the stack the crystals are semiconducting. This indicates that the intermolecular overlap of the wavefunctions is important. Polymers with a delocalized $\pi$-electron would ideally fulfill this condition. 
Many attempts were made to prepare polymers with extended $\pi$-electron systems in the hope that the delocalization of the $\pi$-electrons would generate a high free carrier concentration and high conductivity. But the observed conductivities were low. In the case of polyenes, for example, their optical spectra showed that the threshold for absorption decreases with increasing chain length but tends to saturate at about $2 \mathrm{eV}$. Irrespective of the origin of this optical gap whether due to bond alteration (Kuhn 1949) or due to electron correlation (Ovchinnikov et al 1973), the conductivity is expected to remain low since excitation of carriers along a gap of $2 \mathrm{eV}$ is highly unlikely. Under these circumstances it is not surprising that a combination of ingenious preparational techniques and a knowledge of semiconductor and solid state physics was needed to produce a breakthrough in this field leading to the invention and preparation of conducting polymers.

The real boom in the field of electrically conducting polymers began in 1977 when it was demonstrated (Shirakawa et al 1977) that polyacetylene (PA), an intrinsically insulating polymer, becomes highly conducting on treatment with oxidizing (electron acceptor) or reducing (electron donor) agents. The oxidation (and reduction) reactions which induce high conductivity in PA, using the language of semiconductor physics, are termed as doping: $p$-doping in the former case and $n$-doping in the latter. The discovery of highly conducting PA led to a sudden spurt in research activity directed towards the study of new conducting polymeric systems. The instability of PA in air (Aldissi 1984) further intensified this research (on exposure to air, covalent bonds are formed between oxygen and carbon atoms and these bonds lower the conductivity of PA because of their interruption of conjugated double bonds). A significant breakthrough occurred in 1980 (Shacklette et al 1980) with the discovery that poly ( $p$ phenylene) (PPP) could be doped to conductivity levels quite comparable to those in PA. This polymer is the first example of a non-acetylenic hydrocarbon polymer that can be doped with electron acceptors or electron donors to give polymers with metallic properties. This discovery was also important in the sense that it demonstrated the nonuniqueness of PA system and paved the way for the discovery of a number of new conducting polymers. These polymers, though they share many
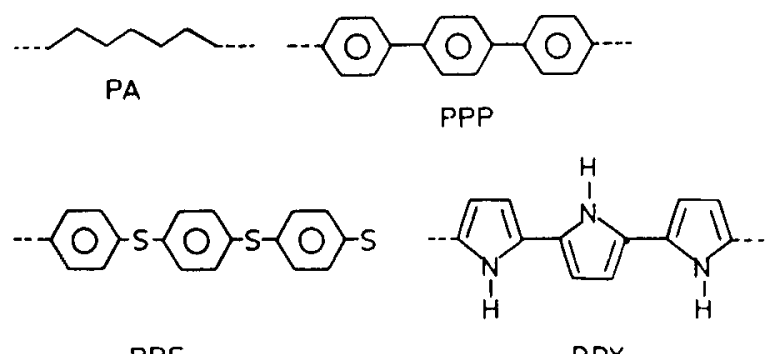

PPS

PPY
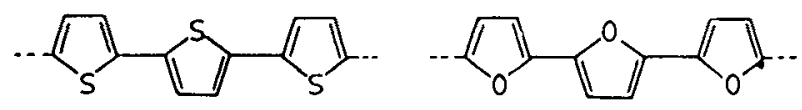

PTP

PFU

Figure 1. Structures of trans-polyacetylene (PA), poly ( $p$-phenylene) (PPP), poly ( $p$-phenylene) sulphide (PPS), polypyrrole (PPY), polythiophene (PTP) and polyfuran (PFU). 
structural similarities (figure 1), have wide range of conductivity depending upon the doping per cent, the alignment of polymer chains and the purity of the sample. At present many such systems are known and they include poly ( $p$-phenylene) sulphide (PPS), polypyrrole (PPY), polythiophene (PTP), polyfuran (PFU) and their derivatives. None of these polymers has however reached the high conductivities of doped PA.

\section{Nature of processes inducing high conductivity in polymers}

Electrically conducting polymers differ from the familiar inorganic crystalline semiconductors such as silicon in two important structural features (Duke and Schein 1980): polymers are molecular in character and lack long range order. The combined influence of molecularity and disorder leads to profound differences between the fundamental physical phenomena that occur in traditional covalent network semiconductors and those that are characteristic of these organic polymers.

The nature of processes which induce high conductivity in polymers is different from those in the inorganic semiconductors. In the doping of inorganic semiconductors, the dopant species occupies positions within the lattice of the host material resulting in the presence of either electron rich or electron deficient sites with no charge transfer occurring between the two sites. The doping reaction in polymers, on the other hand, is a charge transfer reaction. resulting in the partial oxidation or reduction of the polymer, rather than the creation of holes etc. It is now well established (Ferraro and Williams 1987; Scrosati 1988) that the exposure of PA to an oxidizing agent $X$ (or reducing agent $M$ ) leads to the formation of a positively (or negatively) charged polymeric complex and of a counter ion which is the reduced $X^{-}$ (or the oxidized $\mathrm{M}^{+}$) form of the oxidant or reductant. Studies (Hsu et al 1978) based on Raman spectroscopy and Mössbauer spectroscopy show that after exposure to iodine, the PA chain acts as a positively charged polycation counter balanced by $\mathrm{I}_{3}^{-}$or $I_{5}^{-}$ions. Likewise, studies based on optical absorption and ESR spectra indicate that the reduction of PA by exposure to sodium naphthalide in tetrahydrofuran, leads to a negatively charged $(\mathrm{CH})_{x}^{-}$carbanions and $\mathrm{Na}^{+}$counterions (Chung et al 1981). The "doping process" in the case of conducting polymers may be, therefore, more correctly classified as redox processes of the following general scheme.

$$
\text { Polymer }+X=(\text { Polymer })^{n+}+X^{n-} \text {, }
$$

in the case of an oxidation ( $p$-doping) process and

$$
\text { Polymer }+M=(\text { Polymer })^{n-}+M^{n+} \text {, }
$$

for a reduction ( $n$-doping) process.

$$
X=I_{2}, \mathrm{Br}_{2}, \mathrm{AsF}_{5} \ldots \text { and } M=\mathrm{Na} . \mathrm{Li}, \ldots
$$

The above reactions are most likely to occur in the case of unsaturated polymers with $\pi$ electrons as they can be very easily removed or added to the polymeric chains to form polyions and, therefore, these are the types of polymers which assume high conductivity on doping. 


\section{Experimental data on the conductivity of conducting polymers}

\subsection{Dependence on.the conjugation length}

One of the structural features common to all the conducting polymers is the presence of conjugation. How long the conjugated chains must be to allow for high electrical conductivity of the polymers? It has been found that it is the conjugation length of the polymer chain and not its chain length which is important for its high electrical conductivity. The conjugation length of a polymer chain is the average distance between two defects which interrupt the conjugation. Evidently chain ends are such interruptions but there are also others such as $\mathrm{O}=\mathrm{C}-\mathrm{CH}_{2}$.

The effect of conjugation breaking defects on the conductivity is very drastic. Experimentally, it has been found that the conductivity decreases rapidly with decreasing conjugation length. If, for example, the conjugation length is decreased from its pristine value $(100 \mathrm{~A})$ to about $10 \mathrm{~A}$ in the case of trans-PA chains, the conductivity decreases by eight orders of magnitude (Roth et al 1989).

\subsection{Dependence on the doping level}

The conductivity of the conjugated polymers is, in general, found to increase with increase in the doping level of the polymers (figure 2). The doping level of the polymer is determined by the dopant concentration expressed in mol\%. However there is no sharp transition from an insulating to a metallic state. The reasonable analytical fits to the curve in figure 2 in the limit $0.1 \%<y<100 \%$ are

and

$$
\sigma \propto y^{3}
$$

$$
\ln \sigma \propto-y^{-y}
$$

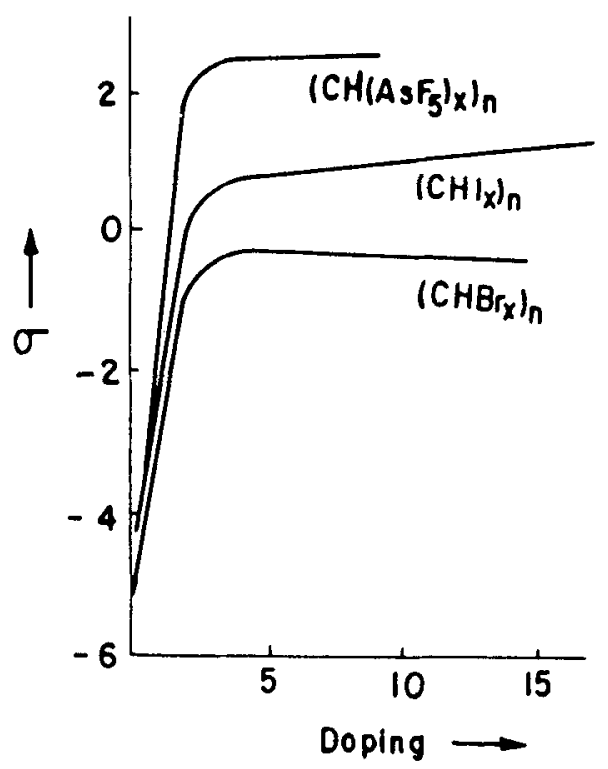

Figure 2. Conductivity change of polyacetylene upon doping. 
where $\sigma$ is the conductivity, $y$ the dopant concentration and $\gamma$ a value between $1 / 4$ and $1 / 2$.

\subsection{Effect of temperature}

In order to understand the effect of temperature on the conductivity of conducting polymers, it is reasonable, first of all, to define two conductivity regimes: the high conductivity regime with conductivity $1000 \mathrm{~S} \mathrm{~cm}^{-1}$ and the modest and low conductivity regime with conductivity $100 \mathrm{~S} \mathrm{~cm}^{-1}$. The vast majority of the conducting polymers belongs to the moderate and the low conductivity regimes. Highly doped PA is the only representative of the high conductivity regime. The temperature dependence of conductivity is different in the two conductivity regimes (figure 3 ). In both the conductivity regimes, the conductivity decreases when the temperature is lowered implying hereby that the temperature coefficient for a synthetic metal is different from that of a metal in which the conductivity increases on cooling the metal. In moderate and low conductivity systems, the conductivity vanishes as the temperature approaches zero whereas in high conductivity systems, the conductivity remains finite (Roth et al 1989).

The temperature dependence of the conductivity also varies with the level of doping (figure 4). For low doped PA samples, for example, the temperature dependence of the

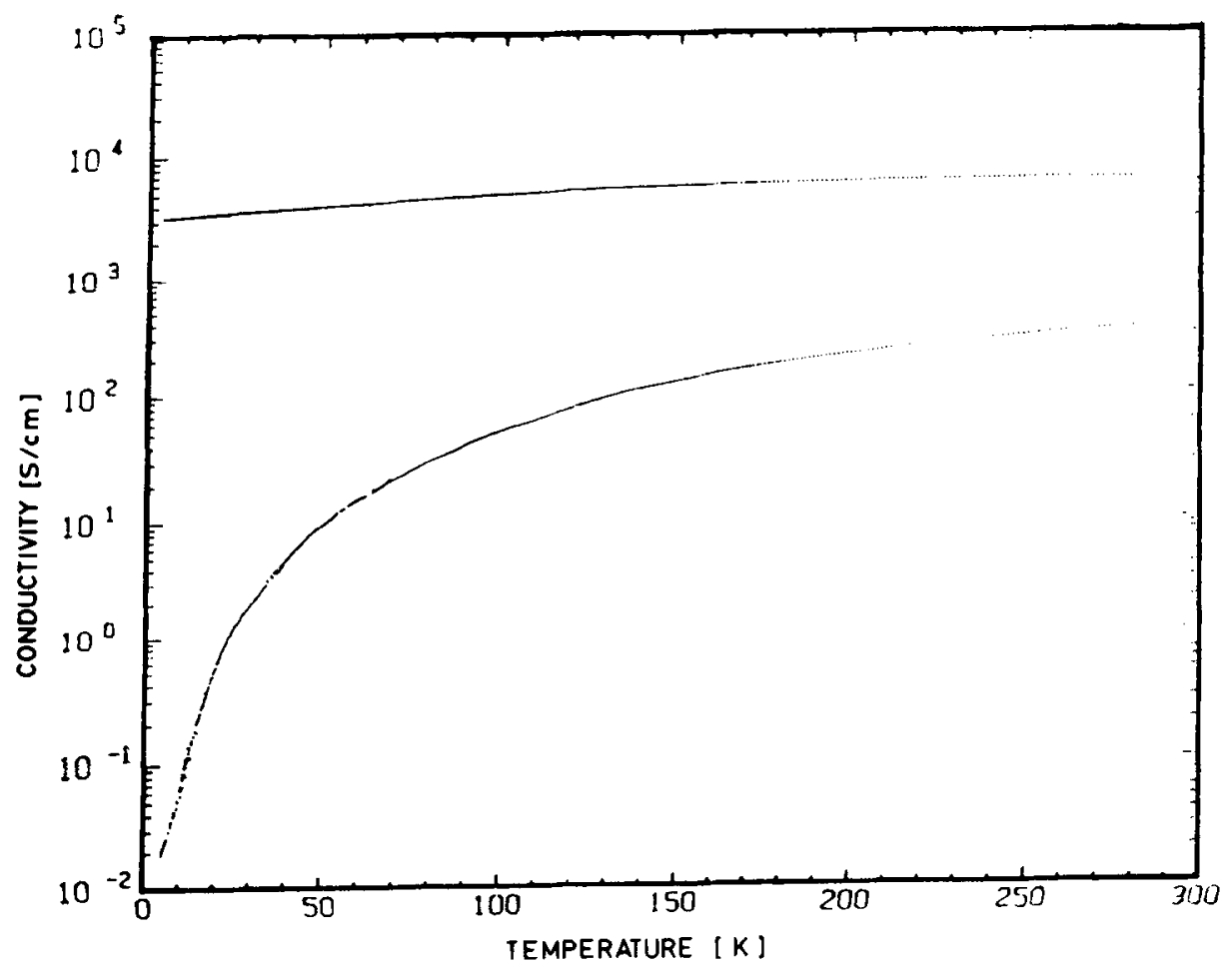

Figure 3. Illustration of the different temperature dependences in the two conductivity regimes. 


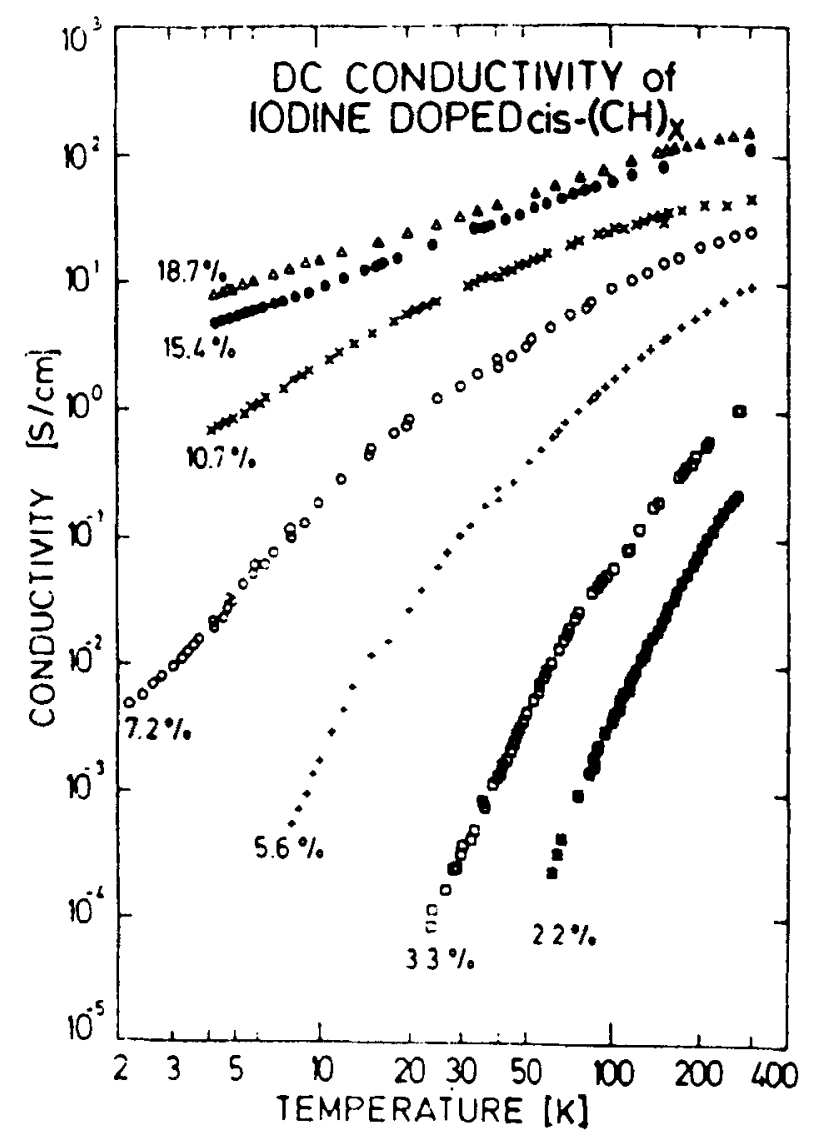

Figure 4. Temperature dependence of a series of iodine doped polyacetylene samples (Schafer et al 1987).

conductivity is very drastic. As the doping level increases, the dependence of the conductivity on temperature becomes less and less and for the most highly doped samples, the conductivity is found to be nearly temperature independent as is the case in dirty metals or disordered alloys. At no stage one observes a typically metallic behaviour i.e. negative temperature coefficient of the conductivity.

Phenomenologically, the observed temperature dependence of conductivity for different levels of doping can be quite well described by

$$
\sigma=\sigma_{0} \exp \left[-\left(T / T_{0}\right)^{-\gamma}\right]
$$

where $\gamma$ and $T_{0}$ are fitting parameters, $\gamma$ depends on the dimensionality $d$ of the hopping process and has values in the range $\frac{1}{4}<\gamma<\frac{1}{2}$. It needs to be noted that (3) above is inspired by Mott's variable range hopping $(\gamma=1$ would correspond to the activated behaviour of a crystalline semiconductor and $\gamma=1 / 4$ to Mott's variable range hopping in three dimensions, typical for amorphous semiconductors) (Mott and Davis 1979). Since this formula also holds good in the case of conducting polymers, it is quite probable that the transport mechanism in these systems is variable range hopping. 


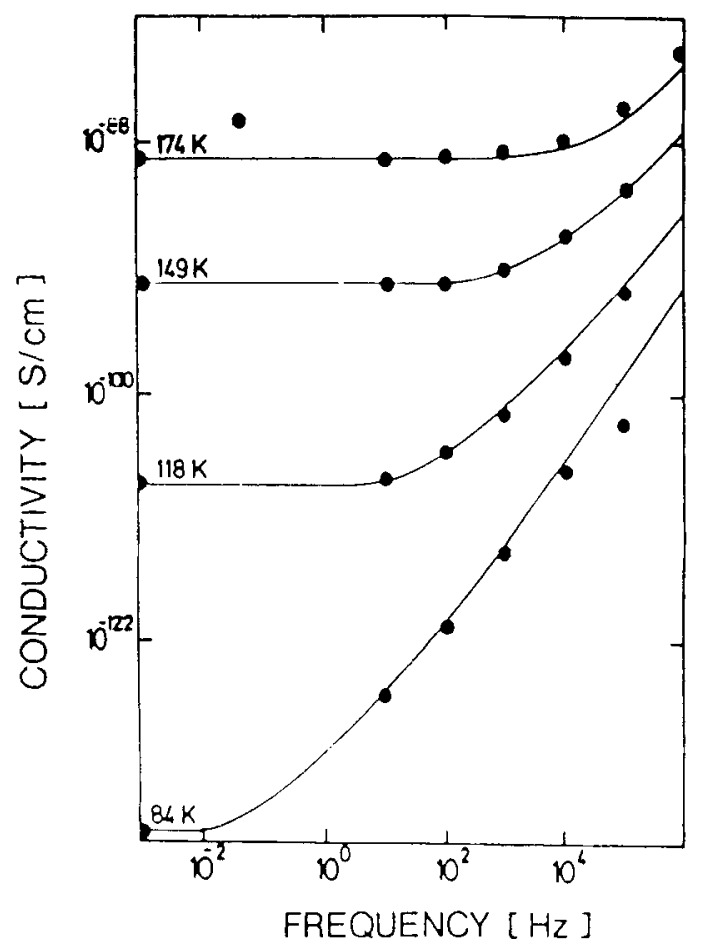

Figure 5. Frequency dependence of the electrical conductivity of very lightly doped polyacetylene at various temperatures (Chroboczek and Summerfield 1984).

\subsection{Frequency dependence}

If the transport mechanism of electrical conductivity is hopping, one would expect a strong frequency dependence of the conductivity. This is due to the fact that electrical conductivity can be decomposed into a dc part and an ac contribution. The dc contribution is frequency independent because in this case the current path will involve several long distant hops which are very unlikely and therefore dc conductivity is very low. In the case of ac contribution, such bottlenecks are not very important because the electric field reverses sign while the electron is waiting to hop. The result is that the ac contribution increases linearly with the frequency. This type of behaviour of electrical conductivity which is characteristic of the disordered materials is also observed in conducting polymers.

In figure 5 is plotted the conductivity of nearly undoped PA versus the frequency at various temperatures. One can see that above a certain threshold frequency, the ac contribution dominates. The strong temperature dependence of the dc part causes also a strong dependence of the threshold on temperature. In doped samples, on the other hand, the dc component of the conductivity is many orders of magnitude higher and the frequency dependence of the total conductivity is revealed only if the experiment is carried out at much higher frequencies.

\section{Nature of charge carriers in conducting polymers}

The nature of charge carriers in conducting polymers is quite different from that in 
inorganic semiconductors. Contrary to inorganic crystalline semiconductors, where charge is transported in general by electrons in the conduction band and holes in the valence band, in doped conjugated polymers charged solitons, polarons and bipolarons act as charge carriers. These quasi-particles arising from a strong interaction between a charge put on the chain (electron, hole) and the molecular structure are the direct consequence of the strong electron-phonon interaction present in these quasione-dimensional polymers. If three dimensional interactions are taken into account, quasi-particles may no longer be stable. In order to have a detailed understanding of the nature of charge carriers and the mechanisms of conduction, the conducting polymers may be divided into two categories: polymers with degenerate ground state and polymers with non-degenerate ground state. The difference between polymers with and polymers without degenerate ground state can be easily visualized if the energy of the two possible structures is plotted as a function of the distortion parameter for a spatially uniform bond arrangement (figure 6). In the case of trans-PA, the energy of two structures is the same whereas for PPP, it is not. In the latter case the quinoid state has a higher energy than the ground state with the more benzenoid (aromatic) bond sequence.

\subsection{Polymers with degenerate ground state}

The degeneracy of the ground state of trans-PA gives rise to the possibility of structural defects (kinks) in chains where there is a change in the sense of bond alternation (figure 7). It is clear from a consideration of bonding of the carbon atoms on the chain that where the defect occurs, a single unpaired electron exists (although the overall charge remains zero) with the result that a new state (energy level) is created at mid gap (i.e. the unpaired electron resides in non-bonding orbital) (figure 8a). This neutral defect state known as soliton is singly occupied and, therefore, has a spin of 1/2 (Rebbi 1979; Heeger et al 1984). The ground state of trans-PA also

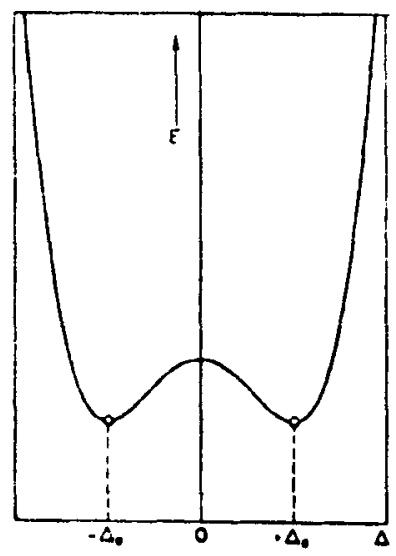

-
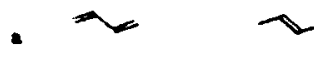

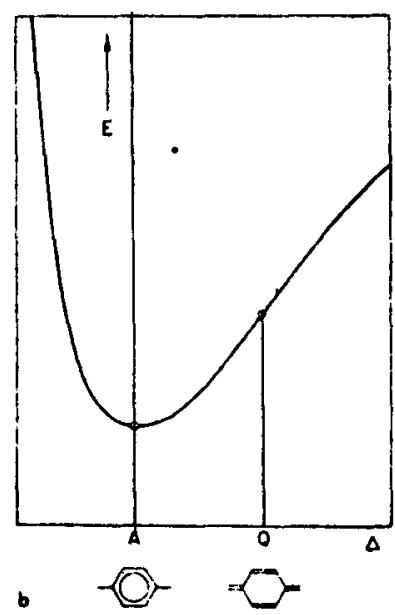

b

Figure 6. Ground state energy as a function of the configuration coordinate for (a) a system with a degenerate ground state such as trans-polyacetylene and (b) a system with nondegenerate ground state such as poly ( $p$-phenylene). 


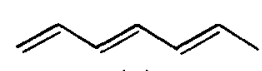

(a)

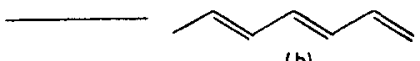

(b)

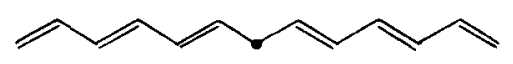

(c)

Figure 7. Degenerate ground state of trans-PA (a and b). PA chain with a defect (neutral soliton).

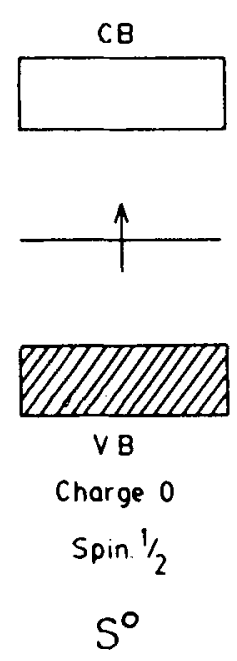

(a)
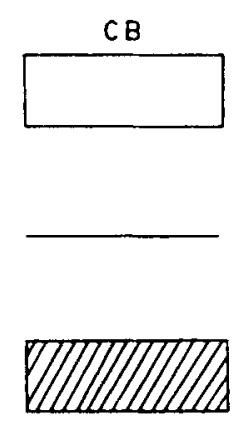

vB

Charge te

Spin 0

$S^{+}$

(b)
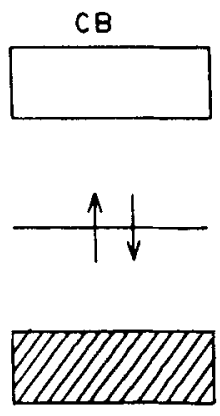

vB

Charge -e

Spin 0

$S^{-}$

(c)

Figure 8. Charge and spin separation in the case of (a) neutral soliton $\left(\mathbf{S}^{0}\right)$, (b) positive soliton $\left(\mathrm{S}^{+}\right)$and $(\mathrm{c})$ negative soliton $\left(\mathrm{S}^{-}\right)$.

already contains a neutral soliton if the chain contains an odd number of electrons. The soliton energy level can accommodate either 0,1 or 2 electrons and thus the soliton may also be positively or negatively charged (figures $8 \mathrm{~b}$ and $\mathrm{c}$ ) giving the unusual property of separating spin and charge (i.e. neutral solitons have spin but no charge, whereas the charged solitons have no spin).

In pure trans-PA there are only neutral solitons on an average of $\sim 1 / 3000$ chain atoms as indicated by the ESR experiments on undoped trans-PA and thus account for the observed paramagnetism in trans-PA (Goldberg et al 1979). When trans-PA is doped this number of neutral solitons is used at an already very low doping level leading to the formation of charged solitons. In the case of defect free trans-PA chain, a charge transfer directly between doping agent and valence and conduction band, respectively will produce an ion radical in the chain i.e. a defect pair instead of an isolated defect. Figure 9 shows the mechanism of p-doping of trans-PA. It can be shown theoretically that a separation of the ion-radical components along the chain into one charged and one neutral soliton corresponds to a higher energy state compared to when the defect pair is held together. This more stable ion-radical pair is called polaron and its formation is accompanied by two energy levels obtained symmetrically above and below the middle level of the band gap. When the doping degree is increased further, i.e. when polaron concentration is increased, the polarons 


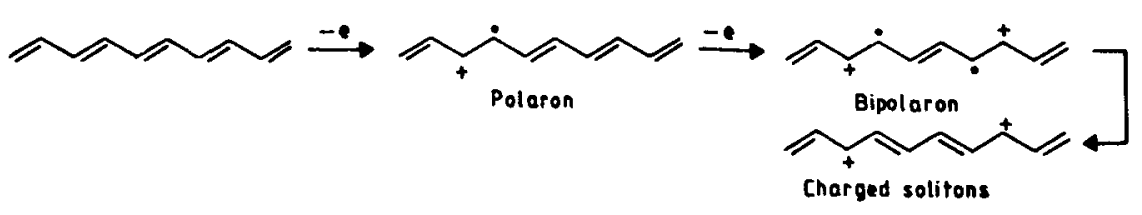

Figure 9. p-doping of defect free trans-PA.

begin to interact with each other. Two polarons interact to give two charged solitons (i.e. two unpaired electrons are united leaving behind two charged solitons) which produce a band half way between valence band and conduction band. A lattice of charged solitons is obtained at about $\sim 4 \mathrm{~mol} \%$ doping.

If one just observes the polarons and ignores their combination process, there occurs in the $p$-doping case, an overlapping between the polaron band and the valence band and the antibonding polaron band and the conduction band at $\sim 5 \%$ doping. The band gap between the polaron levels disappears at about $10 \%$ doping. Since charged solitons are created from polarons, there is a gradual transition from a polaron lattice to a charged soliton lattice (polarons and charged solitons are found to coexist up to a doping level of 3-4\%). Since charged solitons lack spin, low Pauli susceptibility is obtained in the doping range when charged solitons dominate semiconductor metals ( $\sim 1 \%$ doping) to the metal range ( $\sim 10 \%$ doping) (Bredas et al 1982a). At metallic conductivity, the soliton band overlaps the entire band gap between valence band and conduction band so that unpaired electrons with spin can contribute to the conductivity. This occurs at $11 \%$ doping of trans-PA.

\subsection{Polymers with non-degenerate ground state}

In contrast to PA, all other conjugated polymers, including cis-PA, possess nondegenerate ground states and this affects the nature of charge carriers they can support. In such polymers, it has been found that the formation of single solitons, whether as a result of doping or from inherent defects, is energetically unfavourable and the energetically preferred configurations involve paired sites. This can be illustrated by considering an example of polypyrrole (PPY) whose two non-degenerate ground states with an aromatic and quinoid structures are shown in figure 10. Out of these two states, the quinoid structure has a higher energy.

PPY is a poorly conducting material with an energy gap of $3 \cdot 2 \mathrm{eV}$ between the conduction and valence bands. The removal of the first electron in a p-doping (oxidation) process, leads to the formation of a polaron with a relaxation of the structural geometry of the polymers towards a quinoid form extending over 4 pyrrolitic rings (figure 10c). The polaron (1/2 spin) levels are situated at $0.5 \mathrm{eV}$ from the band edges (figure 11a). Calculations (Bredas and Street 1985) have demonstrated that the formation of polaron is energetically most favourable in these conducting polymers (If a pair of radicals exist on a chain, and they are both neutral, they will recombine to eliminate the structural defect (Bredas et al 1982b)). When a second electron is removed from the polymer chain, a bipolaron is formed (figure 10d) which may be defined as a pair of like charges associated with a strong localized lattice distortion, which again extends over 4 pyrolitic rings (it should be noted that this is in contrast to trans-PA where when both defects are charged, they are predicted to 
<smiles></smiles>

(a)<smiles>C=c1ccc(=C2C=CC(C3C=CC(=c4ccc(=c5ccc(=c6ccc(=C)[nH]6)[nH]5)[nH]4)N3)N2)[nH]1</smiles>

(b)

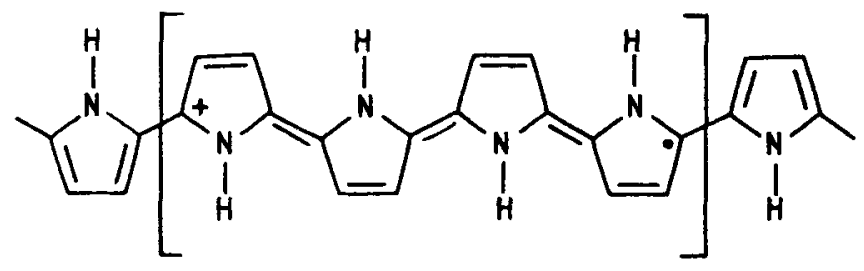

(c)<smiles></smiles>

(d)

Figure 10. (a) and (b) Non-degenerate ground state of polypyrrole (PPY); (c) formation of polaron and (d) formation of bipolaron in PPY as a result of $p$-doping.

separate along the chain). Bipolarons lack spin in contrast to polarons and neutral solitons. The formation of bipolaron is also supported by calculations which show that the formation of one bipolaron is thermodynamically more stable than that of two separated polarons, despite the coulombic repulsion between the two charges confined in the same site (Bredas and Street 1985). Obviously the lattice relaxation is more stronger than that associated with a single polaron and thus the bipolaron levels are moved at $0.75 \mathrm{eV}$ from the band edges (figure $11 \mathrm{~b}$ ). At higher doping levels (which in the case of PPY may extend up to $33 \%$ of dopant mole per polymers repeat unit) the overlap between the bipolaron states leads to the formation within the gap of two bipolaron bands $\sim 0.04 \mathrm{eV}$ wide (figure 10c). It is interesting to note that the band gap 


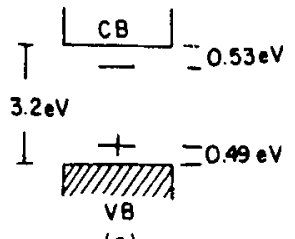

(a)

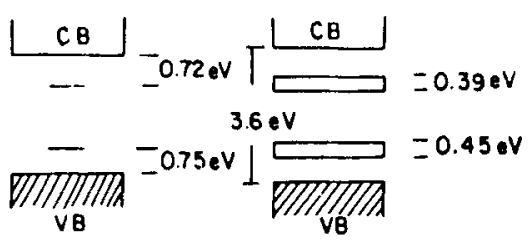

(b)

(c)

$C B=$ conduction band

$\checkmark B=$ volence band

Figure 11. Formation of (a) polaron levels, (b) bipolaron levels and (c) bipolaron bands in PPY as a result of $p$-doping of PPY (Bredas and Street 1985).

in PPY is not completely overlapped even at $100 \%$ doping (hypothetical value) in contrast to trans-PA where the band gap is assumed to disappear completely.

\section{Models for charge transport}

The conductivity of the conjugated polymers increases by many fold on doping as shown above. The conductivity is also found to increase with increase of temperature though the temperature dependence of the conductivity also changes with the doping level of conjugated polymer. All real polymeric samples have in reality a non-ideal structure which further complicates the situation. In PA, for example, the structure may change between trans and cis, resulting in bends, folds, kinks, jogs and similar geometric defects. In PPP or PPY, on the other hand, torsions of monomer units out of their ideal planar position represent another type of geometric disorder. Apart from that, chemical defects are possible, such as cross links between chains, $s p^{3}$ defects in PA and $\alpha$ - $\beta$-linkages of heterocycles in a chain of $\alpha-\alpha^{\prime}$-linked monomer units. Disorder in a polymer chain may also come from doping. At least at low dopant concentrations, the dopant molecules occupy random positions between the chains (Bryant and Glick 1982). The question which arises is how this disorder will influence charge carrier transport by quasi particles in the polymer chain. An element of disorder may either block quasi-particle movement completely by acting as a high barrier, it may attract the quasi-particle and trap it or its influence may only be weak. At present no definite answer is known. It seems reasonable to suppose in the light of the aforementioned facts that no single mechanism of conductivity shall be valid at all temperatures and all doping levels. Various models proposed to explain the charge transport in conducting polymers are briefly discussed below.

\subsection{Variable range hopping model and its modifications}

Mott and Davis (1979) proposed a model to explain the conductivity of amorphous semiconductors. This model is based on the assumption that spatial fluctuations in potential, due to the lack of atomic ordering in these materials, leads to the localization of electronic states. Carriers in localized states can move into adjacent empty states by thermally assisted tunneling called hopping. The short conjugation length along with defects or disorder in conjugated polymers shall also lead to similar localization. Therefore, one may look at conduction in organic conjugated polymers 
as phonon assisted hopping between localized states too. If the temperature is low and if hopping takes place near the Fermi level, Mott's famous variable-range hopping law leads to the following equation for the temperature dependence of the conductivity:

with

$$
\sigma=\sigma_{0} \exp \left(-T_{0} / T\right)^{-1 / 4},
$$

and

$$
T_{0}=24 /\left(\pi r^{3} k N\left(E_{\mathrm{f}}\right)\right)
$$

$$
\sigma_{0}=9 / 4 \sqrt{3 / 2 \pi} e^{2} \gamma_{0}\left(r_{0} N\left(E_{\mathrm{f}}\right) /(k T)\right)^{1 / 2},
$$

where $r_{0}$ is the localization length (decay length of the wave function), $k$, the Boltzman constant, $N\left(E_{\mathrm{f}}\right)$ the density of states near Fermi level and $\gamma_{0}$ the molecular vibration frequency $\left(10^{12}-10^{13} \mathrm{~s}^{-1}\right)$.

Equation (4) does well agree with experimental results, particularly for moderate levels of doping and moderate temperatures. The exponent $-1 / 4$ in this equation is connected with the dimensionality of a charge transport in the solid. If other than the three dimensional charge transport is expected, the exponent changes into $-1 /(n+1)$ with $n$ being the dimensionality.

\subsection{Fluctuation induced tunnelling}

This model of charge transport in conducting polymers is proposed by Sheng (1980). In this model it is assumed that conduction takes place between highly conductive (metal-like) islands separated by potential barriers. These islands are believed to originate from inhomogeneous aggregations of dopant molecules. The charge transfer in this model results from mechanical tunnelling enhanced by thermal fluctuations at higher temperatures. This model leads to

$$
\sigma=\sigma_{0}\left[\exp \left[-T_{1} /\left(T_{1}+T_{0}\right)\right]\right]
$$

with $\sigma_{0}, T_{1}$ and $T_{2}$ being three fitting parameters which depend on the details of the potential barriers between the metal like regions.

\subsection{Interchain-hopping}

The addition of charge in a conjugated polymer leads to the formation of polarons, bipolarons or charged solitons. These quasi-particles are expected to be quite mobile along intact segments of the polymer chain. For electrical transport through real macroscopic samples, however, these quasi-particles have to move finally from one chain to a neighbouring one. How does such inter-chain transfer occur?

The inter-chain transitions of polarons, bipolarons or solitons are difficult to assess because not only a charge but also the molecular distortion has to be transferred. Two models have been proposed: one for bipolaron transitions which is mutatis mutandis also applicable to a pair of confined charged solitons and the other electron hopping between two solitons.

In the model of Chance et al (1984a) the transfer rate for a bipolaron is determined by the combined probability for finding a bipolaron on one chain and a sufficiently long segment free of any excitation on the neighbouring chain (figure 12) and by the 


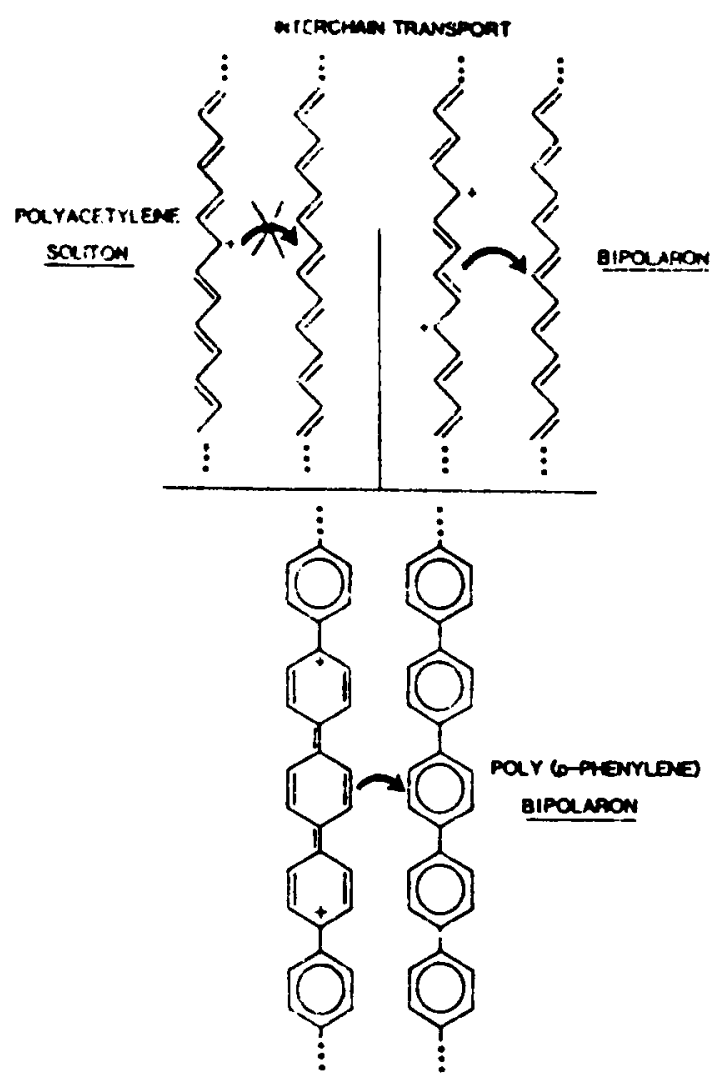

Figure 12. Schematic illustration of interchain transport in polyacetylene and poly ( $p$ phenylene) (Chance et al 1984a).

appropriate Franck-Condon factor $f$. The probability $P_{\mathrm{b}}$ for an inter-chain transfer of bipolaron is given by

$$
P_{\mathrm{b}}=(y / 2)(1-l y / 2)^{1+2},
$$

where $l$ is the length of a bipolaron (expressed in monomer units) and $y$ the molar fraction of dopant molecules. The above relation is valid as long as interactions between bipolarons can be neglected.

Chance et al (1984a) also applied a similar mechanism to the inter-chain transfer of solitons. Two charged solitons on a chain approach each other, being separated by a distance $l$, and perform a transition to an adjacent chain in the same way as for a bipolaron. Taking $\exp (-1)$ for the Franck Condon factor, the total rate is obtained

$$
P_{\mathrm{s}}=y \sum P(l)=\text { const. }\left\{y^{2}(1-y)\right\} /\left\{1-(1-y)^{2} \exp (-\beta l)\right\} .
$$

The transition probability and hence the conductivity as a function of $y$ are s-shaped, levelling off at higher doping concentrations. In this model the hop of two solitons to an adjacent chain is only highly probable if the region of 'wrong' bond arrangement between them is small and only a relatively small molecular distortion has to be transferred together with the charge carrier. This mechanism for a transition is no 
longer possible in doping ranges, at which doping proceeds via conversion of neutral solitons into charged ones and at which the soliton gas is so diluted that only single, charged solitons may be found on the chains. Single solitons are unlikely to hop due to the high energy required for structural reorganization.

To solve the problem for the above doping range, Kivelson $(1981,1982)$ proposed a mechanism for the transition, involving the charged soliton generally pinned to a doping molecule and a neutral soliton on a neighbouring chain. This mechanism involves the tunneling of the unpaired electron of the neutral soliton on one chain to the positively charged soliton at a neighbouring chain, provided the electronic levels of both the excitations nearly coincide. This also requires that the neutral soliton is also close to an ionized impurity, otherwise an energy equal to the binding energy of the charged soliton would have to be overcome. Quantitative analysis of this process gives the following formula:

$$
\sigma(T)=0 \cdot 45 e^{2} /(k T) x_{0}\left(1-x_{0}\right) \gamma(T) /\left(N R_{0}\right) r_{0} /\left(R_{0}\right) \exp \left(-2 \cdot 78 R_{0} / r_{0}\right)
$$

where $x_{0}$ is the fraction of neutral solitons, $\left(1-x_{0}\right)$ the fraction of charged solitons, $N$ the average chain length, $r_{0}$ the average localization length, $R_{0}$ the acceptor distance and $\gamma(T)$ the frequency factor.

The above formula predicts. firstly, a strong exponential increase of the conductivity with doping provided that $x_{0}$ remains constant and second, a power dependence of conductivity on temperature. If three dimensional effects are considered (Baeriswyl and Maki 1989; Vogl et al 1989) this process of inter-soliton hopping becomes unlikely, since chains are bound to one another forming a soliton molecule.

\section{Molecular engineering of conducting polymers}

Currently major efforts in the field of conducting polymers are on the molecular engineering of novel polymers with tailor made conduction properties. This implies even looking for the polymers that are intrinsically good electrical conductors without the need of doping. To achieve this tailoring of conducting polymers, five routes are presently followed.

\subsection{Substitution}

Here one starts with polymers with very small band gap values and then tries to modify their electronic properties by the action of substituents, provided their chemical nature allows substitution reactions. Among the known conducting polymers, trans-PA with a band gap of $1.5 \mathrm{eV}$ (Shirakawa et al 1977) and polythiophene (PTP) with a band gap of $2 \cdot 1 \mathrm{eV}$ (Kobayashi et al 1984) appear to be the ideal starting materials for substitutions. While studying the effect of substituents on polymers, one also needs to take into consideration the steric effects of substituents which are very important and in fact very predominant in most cases. The substituted groups should not be bulky enough to introduce large non-bonded interactions between these groups and thus twist the polymer backbone. Such a twist may lead to non-coplanarity and thus a decrease in the orbital overlap and effective conjugation length, thereby resulting in diminished carrier mobilities and lower conductivities. Further there are 
two important guidelines given below which are of great help in the strategy of substitution. (a) In polymers with degenerate ground state such as trans-PA, the band gap is usually defined in the following way (Mintmire et al 1986):

$$
E_{\text {gap }}=2 \varepsilon_{0}=2\left(\varepsilon_{\mathrm{c}}+\varepsilon_{\mathrm{i}}\right)
$$

where $\varepsilon_{\mathrm{e}}$ is a fixed external contribution to the $E_{\mathrm{gap}}$ due to the $\sigma$ skeleton and $\varepsilon_{\mathrm{i}}$ refers to the internal contribution from the $\pi$ electron framework. In the case of PA, it is now fairly well established that the internal contribution to the gap increases as a function of increasing bond length alternation along the chains and (b) on the other hand, in polymers such as poly ( $p$-phenylene) (PPP), polypyrrole (PPY), polythiophene (PTP) and polyfuran (PFU), with non-degenerate ground state theoretical calculations have shown (Bredas 1985) that in them band gap $\left(E_{\mathrm{g}}\right)$ does not decrease as a function of decreasing bond length alternation as is known in polyacetylene-like compounds but rather as a function of increasing quinoid character of the polymer backbone. What then one needs is a compound in which some quinoid contributions are stabilized in the ground state.

From an analysis (Bakhshi et al 1987) of the Bloch wave-functions of the highest occupied and the lowest unoccupied bands of the $\alpha-\alpha^{\prime}$ linked PPY, PTP and PFU, it is found that the substitution on backbone ( $\beta$-carbon atoms) would influence both ionization potential (IP) and electron affinity (EA) values while the choice of the heteroatom affects EA more than the IP. Using these results as guidelines, there have been studies (Kobayashi et al 1985; Bakhshi and Ladik 1987a,b; Lee and Kertesz 1987, 1988; Nayak and Marynick 1990; Otto and Ladik 1990; Ikenoue et al 1991) of the various derivatives of these systems. While forming derivatives, highly polarizable substituents (such as benzene in polyisothianaphthene (PITN)) are always preferred. High polarizability of the substituents can tend to reduce the electron-electron interactions between the two $\pi$ electrons on the same ring or on neighbouring carbons along the backbone, an effect which would lead to smaller band gap.

\subsection{Ladder structures}

Ladder structures offer another exciting possibility in the search for novel conducting polymers. Among these systems, hydrocarbon polymers with fused aromatic rings have been the focus of enormous interest. This new class of polymers, frequently referred to as one dimensional graphite family, includes members such as polyacene (PAc), polyacenacene (PAcA), polyphenanthrene ( $P P h)$, polyphenanthrophenanthrene ( $P P h P)$ and polyperinaphthalene (PPN). The structures of these systems are shown in figure 13. Except PPN, these members can be considered to form two series of polymers derived from trans-PA and cis-PA respectively. PAc and PAcA can be considered to be the laddered versions of two and three chains of trans-PA respectively while $\mathrm{PPh}$ and $\mathrm{PPhP}$ those of the corresponding cis-PA chains. One may continue building these series further: every higher member in a series has a larger carbon content ultimately leading to 1-D graphite. PPN, on the other hand, can be considered to be a fused version of planar poly (p-phenylene) and cis-PA skeletons as shown in figure 13. Another fusion of cis-PA to PPN shall lead to poly (peri-anthracene) and so on.

There have been many theoretical investigations (Bredas et al 1982a, b; Tanaka et al 1983; Bozovic 1985; Bakhshi and Ladik 1989; Kertesz et al 1989) of the electronic 


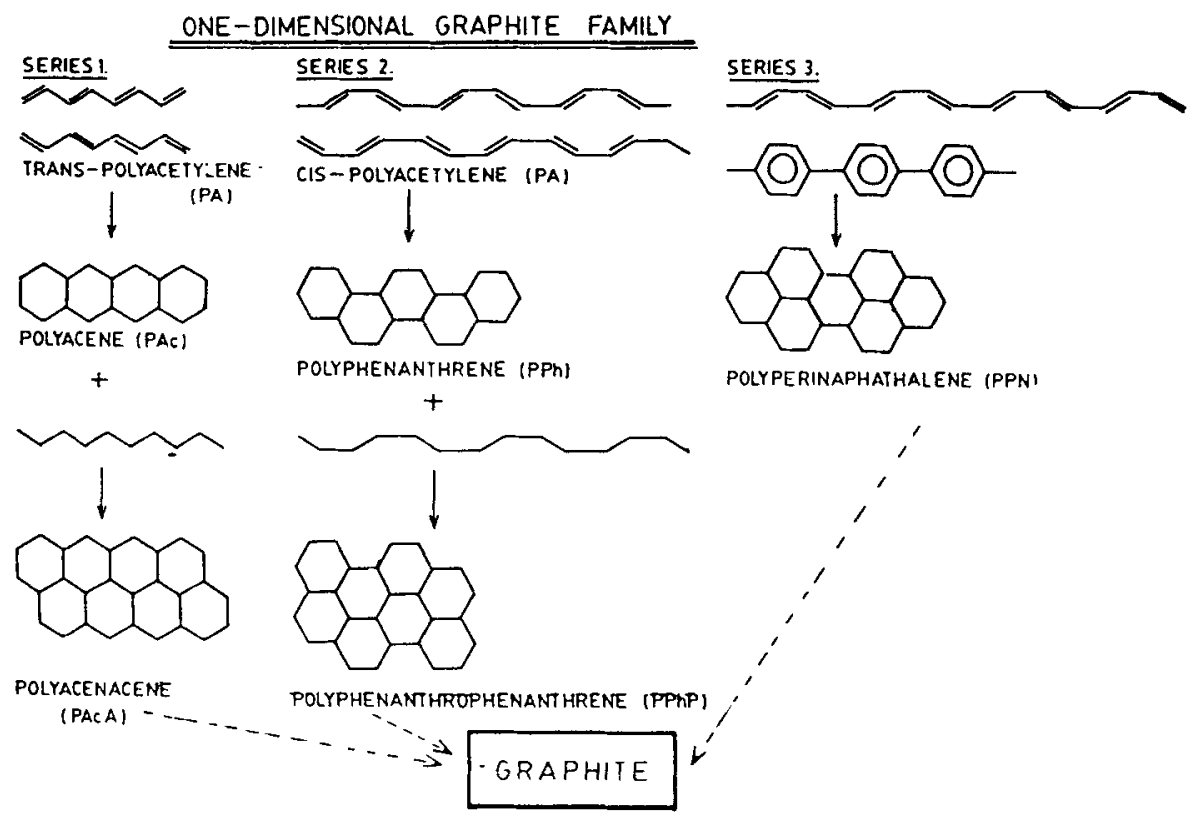

Figure 13. Various members of the one-dimensional graphite family.

structure and conduction properties of the members of 1-D graphite family. PAc is the most studied polymer of this family followed by $\mathrm{PPN}, \mathrm{PPh}$ and $\mathrm{PPhP}$ respectively. The band structure calculations (Bakhshi and Ladik 1989) of PPN show that due to the internal symmetry present in it, its band structure can be considered to be derived from the interaction of two cis-polyacetylene (PA) chains. This interaction splits the original cis PA HOCO and LUCO resulting in a much smaller band gap than in cis PA.

In the case of ladder polymers formed from trans-PA the predicted order of conductivity is polyacenaacene $>$ polyacene $>$ trans-PA. In contrast, in the case of cis-PA ladder polymers, our results show that the band gap of polyphenanthrene (PPh) increases in contrast to that of cis-PA chain (Bakhshi 1992a). These results are interesting in the sense that though PAc and PPh differ from each other only in the mode of growth of condensed aromatic rings, the band gap of PAc is very small as compared to that of PPh. However, as one goes from PPh to polyphenanthrophenanthrene (PPhP), the band gap is again found to decrease (Bakhshi and Ladik 1994).

\subsection{Topological methods}

The electronic properties of fused ring polymers are found to strongly depend on the particular way the rings are fused and the recognition of this has led to the employment of topological methods for estimating band gap relations. These methods are based on the concept of topomers (Polansky and Zander 1982; Gutman and Polansky 1986). It means that one has to construct the corresponding oligomers of a pair of topomers as S- and T- topomers (figure 14) (Liegener et al 1992). These are defined by building them from two identical subunits connected by two bonds. In the S-topomer, 

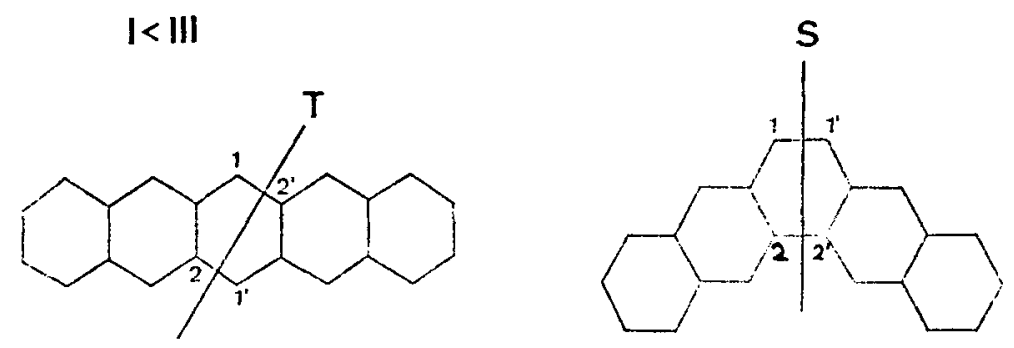

III $<$ II
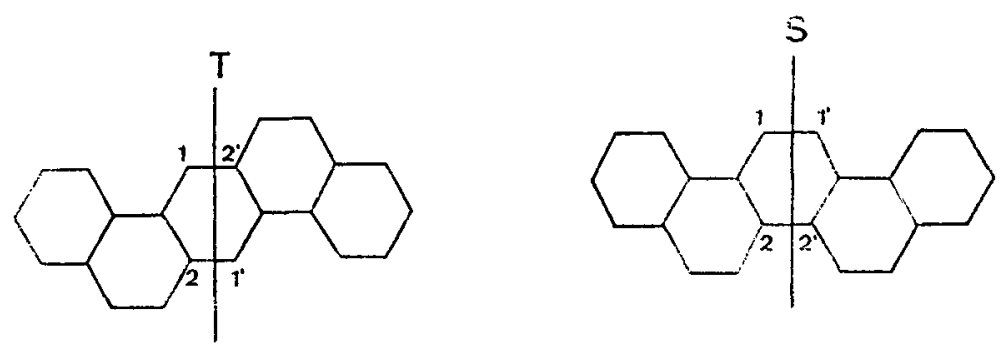

Figure 14. Representation of the oligomers of the polymers: I (polyacene); II (polyphenanthrene) and III (polybenzanthracene) as S- or T-topomers of the corresponding subunits. The pairs of equivalent points are denoted as $\left(1,1^{\prime}\right)$ and $\left(2,2^{\prime}\right)$ where the prime is used to distinguish between the subunits.

the two bonds connect pairwise topologically equivalent atoms while in the $T$ topomer, the end points of the two bonds are interchanged in one subunit. If, for example, A is the T-and B the S-topomer of the same subunit, then the following relations which are the consequences of the interlacing theorem are valid for this pair:

(i) The ionization potential (IP) of $A$ is smaller than the IP of $B$.

(ii) The electron affinity (EA) of $A$ is smaller than the EA of $B$.

(iii) The fundamental band gap of $A$ is smaller than the gap of $B$.

Point (iii) follows immediately from (i) and (ii) because $\Delta=$ IP - EA. The gap of $A$ is, therefore, located inside the gap of B on energy scale.

The above topological arguments have recently been used to rationalize the large differences in the electronic properties of fused ring polymers polyacene, polyphenanthrene and polybenzanthracene (Liegener et al 1992) and in the search for novel low band gap conjugated polymers (Bakhshi et al 1993).

\subsection{Polymeric superlattices}

The fourth possible route to obtain novel conducting polymers is through the technique of growing quasi one dimensional superlattices (copolymers) of conducting polymers. The polymeric quasi one dimensional superlattices (or copolymers) can have tailor made properties depending upon the choice of two semiconducting components, their relative amounts and their arrangement in the polymer chain. It is already well known that copolymerization (Bakhshi 1990 and references therein) 
(periodic or random) considerably influences the electronic properties which however always remain intermediate between those of its constituent homopolymers. Depending upon the band alignment of the two constituent polymers, polymeric superlattices like the inorganic superlattices may be divided into four types (Smith and Maithio 1990) viz. type I, type II - staggered, type II - misaligned and type III (figure 15). Type I applies to such systems where the energy gap of one component is contained entirely within the band gap of the other component. Systems in which the top of the valence band of one component lies within the band gap of the other and the bottom of the conduction band of the second lies in the band gap of the first belong to type II - staggered while in type II - misaligned superlattices, the band match up is such that the conduction band minimum of one is below the valence band maximum of the second component. In type III polymeric superlattices, one component is semimetallic while the other is a normal semiconductor.

The electronic DOS of the various periodic and random quasi one dimensional model superlattices (copolymers) of the type $(\mathrm{Am} \mathrm{Bn})_{X}$ belonging to the class of type I and type II - staggered superlattices have been recently calculated (Bakhshi 1992b). For each of these two types of superlattices, the trends in their electronic structure and conduction properties as a function of (i) composition $(m / n)$, (ii) block sizes $m$ and $n$ and (iii) arrangement of blocks in the copolymer chain have been investigated. It has

(o)

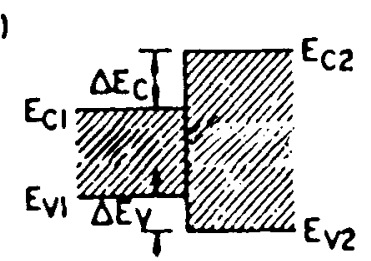

(b)

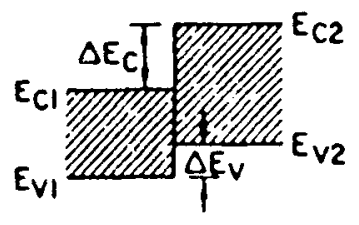

(c)

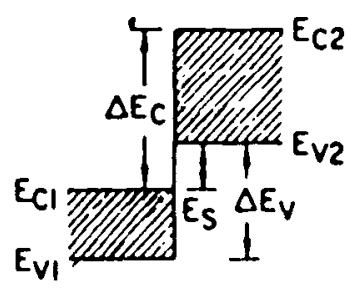

(d)

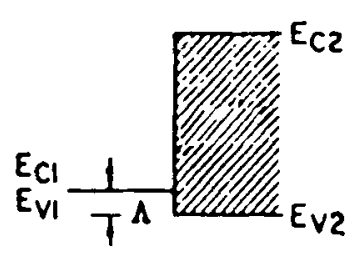

TYPE I

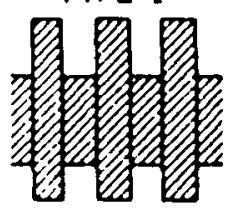

TYPE I-STAGGERED

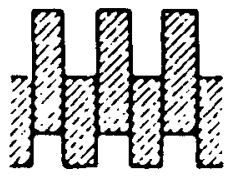

TYPE Z - MISALIGNED

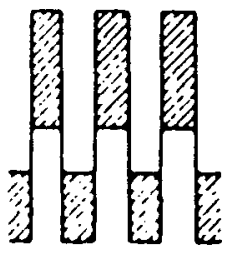

TYPE II

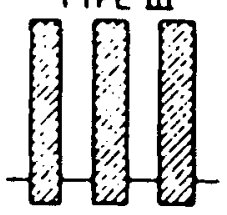

Figure 15. Various types of superlattices ciassified on the basis of the band alignments of the two components $A$ and $B$. 
been found that besides some trends characteristic of each type of superlattice, in both the cases, the tuning of the electronic properties intermediate between those of its components is easier by synthesizing periodic copolymers. Random copolymers, on the other hand, lead relatively faster to the saturated electronic properties characteristic of the lower band gap component and largely independent of the larger gap component. Random copolymers, in general, are also found to be less insulating than the corresponding periodic copolymers.

The above general conclusions are in agreement with the conclusions drawn from the study of the two types of superlattices: type $I$ (consisting of $A=$ furan and $B=$ thiophene) (Bakhshi 1992c) and type II-staggered (consisting of $A=$ pyrrole and $\mathrm{B}=$ vinylene) (Bakhshi and Lovleen 1994).

\subsection{Polymers with electron-withdrawing and electron-donating groups}

Recently a new route to design and synthesize small band gap polymers has been proposed by Havinga et al (1992). In this route one brings together electron-donating and electron-withdrawing groups along a conjugated backbone. They have been able to synthesize polysquaraines and polycroconaines with a band gap of $0.5 \mathrm{eV}$ using this concept. Using the same route, Lambert and Ferraris (1991a, b) have synthesized polydicyanomethylene-cyclopenta-dithiophene (PCNTh), a polymer made by the succession of bithiophene units in which the two rings are linked by a dicyanomethylene group. The experimental band gap of this polymer, as obtained from absorption<smiles>Cc1cc2c(s1)-c1sc(Cl)cc1C2=C(C#N)C#N</smiles>

(a)<smiles>N#CC(C#N)=C1c2cc([Tl])[nH]c2-c2[nH]c([Tl])cc21</smiles>

(b)

Figure 16. Structures of the unit cell of (a) polydicyanomethylene-cyclopenta-dithiophene (PCNTh) and (b) polydicyanomethylene-cyclopenta-dipyrrole (PCNPy). 
spectra, is $\sim 0.80 \mathrm{eV}$. Toussaint and Bredas (1993) have recently theoretically analysed the geometric and electronic structures of PCNTh and its nitrogen analogue PCNPy (figure 16). Their results show that the introduction of a withdrawing group between the two thiophene or pyrrole rings of the unit cell induces major geometry differences within the rings resulting in a geometric structure that can be viewed as a combination of aromatic and quinoid geometries.

\section{Applications}

Stability and processing characteristics have been the main barriers to the commercialization of the conducting polymers. Stability pertains primarily to reactivity towards oxygen or moisture. Doped PA, for example, is very reactive towards oxygen and moisture-it rapidly suffers irreversible loss of conductivity in the atmosphere. Some other polymers such as polypyrrole, polythiophene, polyaniline, polyphenylene vinylene exhibit much greater stability toward ambient conditions. While not as conductive as PA, they do well enough for many applications.

The second key issue in conducting polymer technology is ease of processing. The first conducting polymers were hard to process because of their insolubility and infusibility. But considerable progress has been made in the past few years (Aldissi 1993). Several conducting polymers can now be prepared as dispersions and applied as coatings while others can be prepared from processible precursor polymers. Many of the polymers can now be blended with conventional thermoplastics such as polyethylene or polyvinylchloride to produce a partially conducting material that is readily processed.

\subsection{Light weight and rechargeable batteries}

The most commonly proposed application is their use as electrodes in light weight and rechargeable batteries. In polymers where both $p$ - and $n$-doping processes are feasible, the possibility exists of their use as both positive and negative electrodes in the same battery system. This concept was exploited in the early 1980's by Mac Diarmid and Heeger, in the case of polyacetylene (Nigrey et al 1979; MacInnes et al 1981). Their battery has the following configuration in its fully discharged state (figure 17):

$$
(\mathrm{CH})_{x} / \mathrm{LiClO}_{4}-\mathrm{PC} /(\mathrm{CH})_{x}
$$

Compared to ordinary batteries, PA battery is not only lighter in weight but also has both higher energy and power densities. The polymer electrodes of these batteries have a longer life than the metal electrodes of the ordinary batteries because the ions involved in the delivery and storage of charge come from the solution rather than from the electrodes themselves and therefore these electrodes are saved from the mechanical wear brought about by the dissolution and the redeposition of electrode materials that takes place during charge-discharge cycles in ordinary batteries. Another advantage of the polymer batteries is the absence of toxic materials in them and therefore the disposal problems are minimized. The invention of the polymer batteries has been heralded as the invention that would make electric car a reality as 


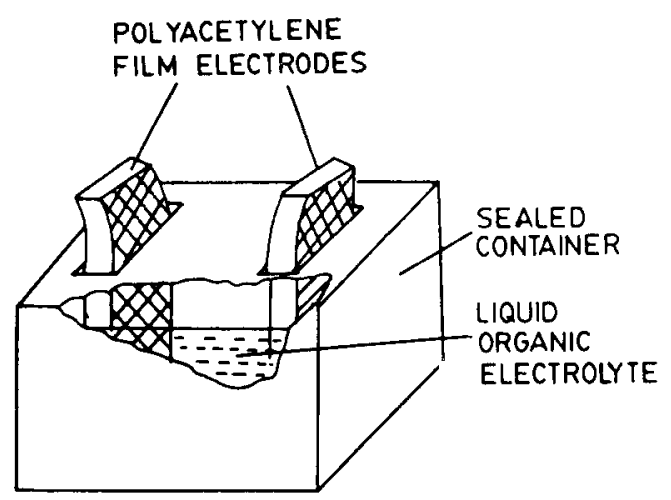

Figure 17. Scheme of a battery using two polyacetylene electrodes and an organic electrolyte formed by a solution of lithium perchlorate in propylene carbonate $\left(\mathrm{LiClO}_{4}-\mathrm{PC}\right)$.

the light weight polymer batteries would not weigh a car down to the extent as the heavy lead acid batteries would do.

The Bridgestone Corporation of Japan have developed coin type rechargeable polymer lithium batteries with a conducting polymer polyaniline and the high capacity lithium aluminium alloy as the two electrodes. The characteristics of these batteries are low self discharge, high voltage and long-term reliability. One of the unique features of this rechargeable polymer lithium battery is that it can be used as a power source in combination with solar cells. This feature opens up many new possibilities for application as a power source. The operation of electronic clock, watches, calculators, remote control devices of audio-visual units etc stand to benefit from this technology.

\subsection{Solid state batteries}

Recently batteries with a full solid state configuration have also been realized using the electrically conducting polymers (Scrosati and Owens 1987). The absence of liquids or gases in the solid state cell leads to high durability and reliabilityproperties which may become precious when safety is essential. Further the solid state batteries from the thin films of conducting polymers may provide plasticity-a feature which would be highly welcome in various applications.

A solid state $\mathrm{Li} / \mathrm{I}_{2}-\mathrm{PA}$ battery has been realized by placing into a direct contact a lithium disk with an iodine-doped PA film (figure 18). Contact between lithium and iodine doped PA brings about immediately a reaction with the formation of lithium iodide.

$$
2 x \mathrm{Li}+\mathrm{CH}\left(\mathrm{I}_{2}\right)_{y x} \rightarrow 2 x(1-n) \mathrm{Li}+\mathrm{CH}\left(\mathrm{I}_{2}\right)_{y-n x}+2 x n \mathrm{Lil} .
$$

Under open circuit conditions, this reaction takes place until the formation of uniform layer of lithium iodide which acts as a electrolyte.

\subsection{Electrochromic devices}

The doping processes of certain conducting polymers are accompanied by changes in 


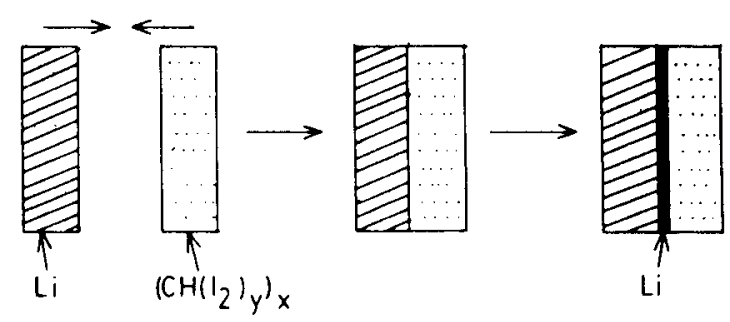

Figure 18. Formation of a lithium polyacetylene solid state battery (Scrosati and Owens 1987).

the colour. This effect has been conveniently exploited in the realization of electrochromic display (ECD) devices. An ECD device changes colour on the application of an electric potential. Thin films of a conducting polymer polythiophene, for example, are red in the doped state and deep blue in the undoped state. However the fact that a material changes colour is not sufficient to permit its use in a display system, its switching time between these two states must be very short and it should maintain these properties in spite of intensive use. In the case of polythiophene, switching time around 30 millisec and a stability exceeding one million doping/undoping cycles have been measured. This value corresponds to about 2 years of operation for a frequency of one cycle per min. Some of the applications of the ECDs include time tables in airports and train stations, calculators, computers, clocks and any other piece of equipment that utilizes a liquid crystal display. The electrochromic display devices have several advantages over the liquid crystal display devices. The ECD have no limited visual angle, may be constructed in large dimensions and have optical memory since the colour acquired remains also after the driving voltage pulse has been removed. The disadvantage is mainly associated with the comparatively slow response time of ECD's since the driving mechanism deals with ions which generally have a low diffusion coefficient in polymers. The principle of electrochromic devices could also be exploited in ordinary window glass. Very thin polymer layers embedded in a colourless solid electrolyte and sandwiched between two layers of glass, may tint a window when an electric potential is applied. The degree of tinting can be controlled by the size of the electric potential. Conducting polymers have the ability to absorb electromagnetic energy at low frequencies and could therefore be used as shields against electromagnetic interference.

\subsection{Sensors}

Sensors are used for detecting and measuring the concentration of various chemical species in liquid or gas phase. An "ideal" chemical sensor should exhibit high sensitivity, selectivity, high operation speed, reversibility and stability under operating conditions. Moreover it should not be sensitive to temperature changes or radiations of various kinds. Conducting polymers with conjugated $\pi$-bonds are likely to show properties meeting the above requirements because their conductivity depends on the electronic structure which can undergo changes under the influence of a chemical species adsorbed on the surface of the polymer layer. These changes may result from e.g. redox or acid-base interactions between polymer and the chemical species. 
PA may be, for example, used for determining the concentration of nitrate ions in acid solutions because as a result of the intercalation oxidation, the conductivity of the polymer changes. The usability of PPY and its derivatives for various gas sensors has also been investigated. Recently the use of conducting polymers for preparing enzymatic electrochemical microsensors sensitive to glucose content has been reported. Conducting polymers such as polyfuran and polythiophene are also expected to have profound uses in humidity sensors and radiation detectors as the conductivity of these doped polymers varies considerably when exposed to humidity or radiation.

\subsection{Microelectronics}

The fact that many conducting polymers are semiconductors in the undoped or lightly doped state has prompted proposals for their use in microelectronics. Both industrial and academic groups are pursuing their use in semiconductor devices and especially field effect transistors (FETs) (Burroughes and Friend 1988; Burroughes et al 1988; Friend 1993). For a FET's active layer - the semiconducting layer between the source and drain electrodes-micrometer thick films are required. Advances during the last few years have made it possible to prepare as thin as this from the polymers with standard techniques such as spin coating and thermal evaporation. The FET in figure 19, for example, is prepared by spin coating a solution of a precursor polymer on a substrate having the required electrode pattern. To convert it into a conducting polymer, the precursor was heated in a dilute stream of hydrogen chloride gas. The carrier mobility of $10^{-6}$ to $10^{-4} \mathrm{~cm}^{2} / \mathrm{V}-\mathrm{s}$ in such FET's is however several orders of magnitude below the magnitudes of a silicon based device restricting the use of polymeric FET's to relatively low frequencies. More recent work has improved these mobilities to $10^{-1} \mathrm{~cm}^{2} / \mathrm{V}-\mathrm{s}$. This development shall soon open the way to uses such as flat-panel colour displays for computers and possibly flat colour televisions when tied to a liquid crystal matrix.

Light emitting diodes (LED's) have also been fabricated from conjugated polymers using poly ( $p$-phenylene-vinylene) as the emissive layer (Friend 1993; Burroughes et al 1990) (figure 20). Flexible, almost entirely metal free LED's have been developed (Gustavsson et al 1992) and such feats have been made possible by among other reasons, the advances in the chemistry of "processible conjugated polymers" over the past several years and the focussed work on the physics of electroluminescence in these materials. These polymer based LED's could be competitive in display applications in

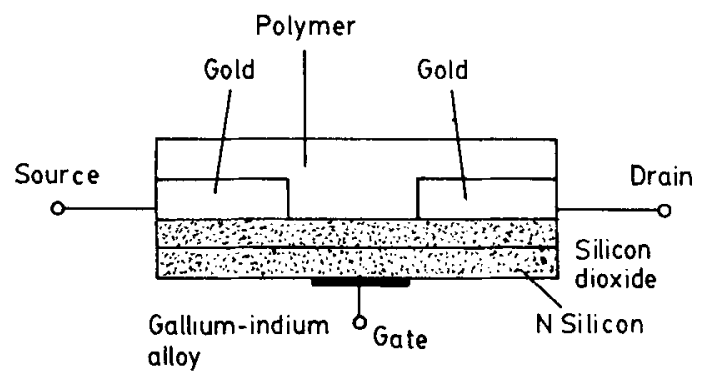

Figure 19. Schematic representation of a field-effect transistor containing an active thin film layer of a conducting polymer. 


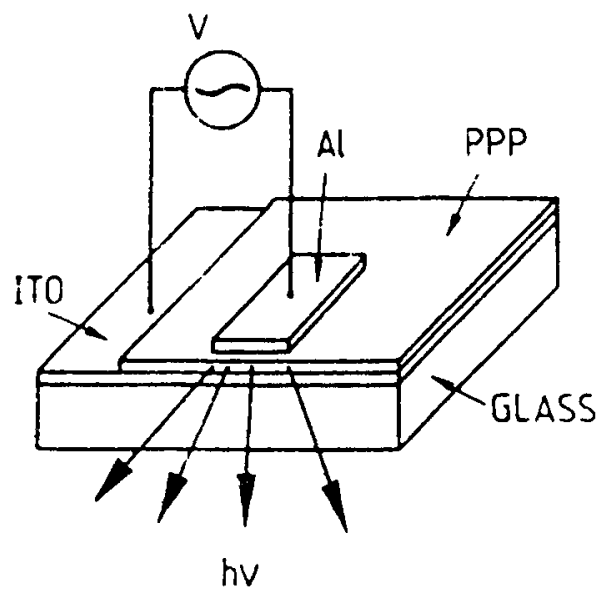

Figure 20. Schematic setup of a light emitting device based on poly ( $p$-phenylene) (Gram et al 1992).

the future because of the potential ease, low cost of fabrication and large surface area of devices based upon processible polymers.

\subsection{Other applications}

Electrically conducting polymers which can be processed thermoplastically and possess the advantageous mechanical behaviour and corrosion stability of plastics would open up an entirely new field of applications. Cables for conducting electricity, wall papers which allow electrical heating, new materials for antistatic equipment are only some few fields in which electrically conducting polymers would find a big market. The polymerization and doping of conducting polymers on such fabrics as nylon, cotton and polyester to produce fabrics with a range of conductivities under the trade name Contex has been achieved by Millikaen and Co., Spartanburg, S.C. These materials are under intense study for use in future aircraft and tanks. They could also be used for heated clothing, shielding computers against electromagnetic interference, static dissipation in high speed missiles and conveyor belts that handle static-sensitive electronic and flammable articles/polymers. Electrically conducting polymers are also regarded as candidates of high potential for passivating layers with respect to photocorrosion of photoelectrodes. Films of polypyrrole have already been tested as coatings for certain semiconductor electrodes.

Other speculative applications of these conducting polymers include such diverse areas as solar cells and drug delivery systems for the human body. Although conducting polymers can absorb sun light, their conversion efficiencies fall far below those required for effective solar cells. Their potential in drug delivery depends on the redox properties of a polymer implanted in the body. If a drug molecule could function as a dopant ion, the drug would, in principle, be released on dedoping the polymer by application of a small electric potential. In practice many chemical and medical problems will have to be solved before experiments can begin in vivo. Structures similar to muscle fibres have been proposed by researchers in Italy and Japan. They are based on the property that a conducting polymer in a fibre form undergoes 
dimensional changes as a result of electrochemical doping and undoping. Work results from expansion and contraction along the fibre's length. More possible applications include conductive paints, toners for reprographics and printing, and as components for aircraft where the combination of light weight, mechanical strength and moderately high conductivity are required.

\section{References}

Aldissi M ed. 1993 Intrinsically conducting polymers: An emerging technology (Dordrecht: Kluwer Academic Publishers)

Aldissi M 1984 Synth. Metals 9131

Baeriswyl D and Maki K 1989 Synth. Metals 28 D507

Bakhshi A K. Ladik J and Seel M 1987 Phys. Rev. B35 704

Bakhshi A K and Ladik J 1987a Solid State Commun. 6171

Bakhshi A K and Ladik J 1987b Solid State Commun. 631157

Bakhshi A K and Ladik J 1989 Synth. Metals 30115

Bakhshi A K 1990 J. Mol. Struct. (Theochem) 209177

Bakhshi A K 1992a Superlattices \& Microstructure 11465

Bakhshi A K 1992b Superlattices \& Microstructure 11473

Bakhshi A K 1992c J. Chem. Phys. 962339

Bakhshi A K, Liegener C M and Ladik J 1993 Chem. Phys. 17365

Bakhshi A K and Ladik J 1994 Indian J. Chem. A33 494

Bakhshi A K and Lovleen 1994 Superlattices \& Microstructure 13437

Bozovic I 1985 Phys. Rev. B32 8136

Bredas J L, Chance R R, Baughman R H and Silbey R 1982a J. Chem. Phys. 763673

Bredas J L, Chance R R and Silbey R 1982b Phys. Rev. B70 1132

Bredas J L 1985 J. Chem. Phys. 823808

Bredas J L and Street G B 1985 Acc. Chem. Res. 18309

Bryant G W and Glick A J 1982 Phys. Rev. B26 5855

Burroughes J H, Jones C A and Friend R H 1988 Nature 335137

Burroughes J H and Friend R H 1988 Physics World 124

Burroughes J H, Bradley D D C, Brown A R, Marks R N, Mackay K, Friend R H, Burn P L and Holmes A B 1990 Nature 347539

Chance R R, Bredas J L and Silbey R 1984a Phys. Rev. B29 4491

Chance R R, Boudreaux D S, Eckhardt H, Elsenbaumer R L, Frommer J E, Bredas J L and Silby R 1984b Quantum chemistry of polymers - solid state aspects 221

Chroboczek J A and Summerfield S 1983 J. de Phys. 4429

Chung T C, Feldblum A, Heeger A J and MacDiarmid A G 1981 J. Chem. Phys. 745504

Duke C B and Schein L B 1980 Physics Today 3342

Ferraro J R and Williams J M 1987 Introduction to synthetic electrical conductors (Orlands: Academic Press) p. 126

Friend R H 1993 in Conjugated polymers and related materials: The interconnection of chemical and electronic structure (eds) W R Salaneck, I Lundstrom and B Ranby (Oxford: Oxford Scientific) p. 285

Goldberg I B, Crowe H R and Newman P R 1979 J. Chem. Phys. 701132

Gram G, Leditzky G, Ullrich B and Leising G 1992 Adv. Mater. 436

Gustavsson G, Cao Y, Treacy G M, Klavetter F, Colaneri N and Heeger A J 1992 Nature 357477

Gutman I and Polansky O E 1986 Mathematical concepts in organic chemistry (Berlin: Springer)

Havinga E E, ten Hoeve W and Wynberg H 1992 Polym. Bull. 29119

Heeger T C, Blanchet G, Tung T C and Fincher C R 1984 Synth. Metals 9173

Hsu S L, Signorelli A J, Pez G P and Baugham R H 1978 J. Chem. Phys. 69106

Ikenoue Y, Wudl F and Heeger A J 1991 Synth. Metals 401

Kertesz M, Lee Y S and Stewart James J P 1989 Int. J. Quantum Chem. 35305

Kivelson S 1981 Phys. Rev. Lett. B46 1344

Kivelson S 1982 Phys. Rev. B23 5397

Kobayashi M, Chen J, Chung T C, Moraes F, Heeger A J and Wudl F 1984 Synth. Metals 977 
Kobayashi M, Colaneri N, Boysel M, Wudl F and Heeger A J 1985 J. Chem. Phys. 825717

Kuhn H 1949 J. Chem. Phys. 171189

Lambert T and Ferraris J P 1991 a J. Chem. Soc. Chem. Commun. 752

Lambert T and Ferraris J P 1991b J. Chem. Soc. Chem. Commun. 1268

Lee Y S and Kertesz M 1987 Int. J. Quantum Chem. 21163

Lee Y S and Kertesz M 1988 J. Chem. Phys. 882609

Liegener C M, Bakhshi A K and Ladik J 1992 Chem. Phys. Lett. 19962

Little W A 1964 Phys. Rev. A1341416

MacInnes D Jr, Druy M A, Nigrey P J, Nairns D P, MacDiarmid A G and Heeger A J 1981 J. Chem. Soc. Chem. Commun. 317

Mintmire J W, White C T and Elert M L 1986 Synth. Metals 16235

Mott N F and Davis E A 1979 Electronic processes in non-crystalline materials (Oxford: Clarendon Press) 2nd edition

Nayak K and Marynick D S 1990 Macromolecules 232237

Nigrey P J, MacDiarmid A G and Heeger A J 1979 J. Chem. Soc. Chem. Commun. 594

Otto P and Ladik J $1990 \mathrm{~J}$. Mol. Struct. (Theochem) 36327

Ovchinnikov A A. Ukrainskii II and Kventsel G V 1973 Sov. Phys. Usp. 15575

Polansky O E and Zander M $1982 \mathrm{~J}$. Mol. Struct. 84361

Rebbi C 1979 Sci. Am. 24076

Roth S, Bleier Hartmut and Pukacki Wojciech 1989 Faraday Discuss. Chem. Soc. 88223

Schafer-Siebert D, Budrowski C, Kuzmany H and Roth S 1987 in Electronic properties of conjugated polymers (eds) H Kuzmany, M Mehring and S Roth (Heidelberg: Springer) p. 38

Scrosati B 1988 Prog. Solid State Chem. 181

Scrosati B and Owens B B 1987 Solid State lonics 23275

Shacklette L W, Eckhardt H, Chance R R and Baughman R H 1980 J. Chem. Soc. Chem. Commun. 854

Shirakawa H, Louis E J, MacDiarmid A G, Chiang C K and Heeger A J 1977 J. Chem. Soc. Chem. Commun. 578

Sheng P 1980 Phys. Rev. B21 2180

Smith D L and Maithio C 1990 Rev. Mod. Phys. 62173

Tanaka K, Ohzeki K, Nankai S and Vamabe T 1983 J. Phys. Chem. Solids 441069

Toussaint J M and Bredas J L 1993 Synth. Metals 61103

Vogl P. Campbell D K and Sankey O F 1989 Synth. Metals 28 D513 